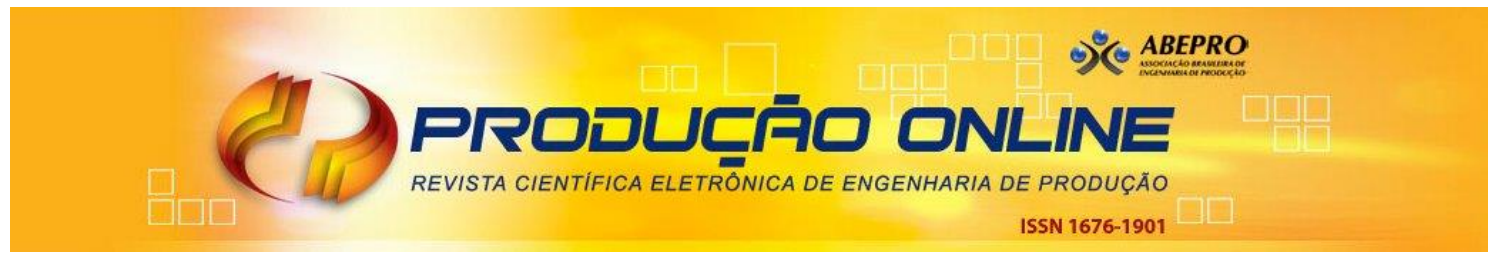

\title{
DISTÚRBIOS OSTEOMUSCULARES AUTORREFERIDOS ENTRE OS TRABALHADORES DA LIMPEZA URBANA ${ }^{1}$
}

\section{MUSCULOSKELETAL DISORDERS SELF-REPORTED BETWEEN URBAN CLEANING WORKERS}

\author{
Milena Nunes Alves de Sousa* ${ }^{* *}$ E-mail: minualsa@hotmail.com \\ Kamilla Gualberto Ferreira** E-mail: kamilla.gualberto@gmail.com \\ Débora Araújo Marinho** E-mail: deboraaraujomarinho@hotmail.com \\ Givanete Alves Gomes** E-mail: gilzinha.alves@hotmail.com \\ Yoly Sousa Ramos*** E-mail: yolysramos@hotmail.com \\ Monica de Andrade* E-mail: monicaamorraye@gmail.com \\ * Universidade de Franca (UNIFRAN), Franca, SP \\ **Faculdades Integradas de Patos (FIP), Patos, PB \\ ${ }^{* * *}$ Universidade Federal de Campina Grande (UFCG), Campina Grande, PB
}

\begin{abstract}
Resumo: Objetivou-se avaliar a prevalência autorreferida de distúrbios osteomusculares entre os trabalhadores da limpeza urbana. Foi realizado estudo descritivo, de prevalência com abordagem quantitativa, tendo sido realizado no município de Patos - PB com 38 agentes da limpeza urbana local $(60,1 \%$ do universo de pesquisa). A coleta de dados foi realizada entre os meses de setembro a outubro a partir da aplicação de dois questionários. O primeiro contemplou variáveis sociais, demográficas, profissionais e clínicas dos pesquisados e o segundo foi o instrumento validado denominado de Questionário Nórdico de Sintomas Osteomusculares. Os dados foram analisados no Statistical Package for the Social Sciences, versão 21 e realizou-se estatística descritiva e inferencial (correlação não paramétrica Spearman e a correlação bisserial por ponto). Aceitou-se como estatisticamente significativo um $p \leq 0,05$. Constatou-se que a prevalência autorreferida de sintomas osteomusculares foi de $45,9 \%(n=18)$, haja vista tais trabalhadores relataram a presença de dor, formigamento e dormência no corpo nos últimos 12 meses, com maior acometimento na região lombar $(10,5 \%)$. Também, $15,8 \%(n=6)$ relataram impedimento de realizar atividades normais nos últimos 12 meses em decorrência destes problemas, bem como consultaram profissional de saúde nos últimos 12 meses por problemas osteomusculares e tiveram algum problema osteomuscular nos últimos sete dias. Os achados indicaram significativa prevalência autorreferida de distúrbios osteomusculares entre os agentes da limpeza urbana. O quadro demanda intervenções no campo da promoção da saúde no trabalho.
\end{abstract}

Palavras-Chaves: Limpeza Urbana. Transtornos Traumáticos Cumulativos. Prevalência.

Abstract: The aim of this paper is to evaluate the prevalence of self-reported musculoskeletal disorders among workers in urban cleaning. A descriptive study of prevalence with quantitative approach and was conducted in Patos- PB with 38 agents of local urban cleaning $(60.1 \%$ of the research universe). Data collection was carried out between the months September-October from the application of two questionnaires. The first included social, demographic, and clinical professionals of respondents and the second was validated instrument called Nordic Musculoskeletal Questionnaire. Data were analyzed using the Statistical Package for the Social Sciences, version 21 and held descriptive and inferential statistics (Spearman nonparametric correlation and correlation biserial). It

\footnotetext{
${ }^{1}$ Artigo originado a partir de relatório de pesquisa de pós-doutoramento em Promoção de Saúde pela Universidade de Franca, Franca-SP, Brasil, desenvolvida sem fontes de financiamento, sem conflitos de interesse e aprovado pelo Comitê de Ética em Pesquisa das Faculdades Integradas de Patos, parecer: 1211722.
} 
was accepted as statistically significant at $p<0.05$. It was found that the prevalence of self-reported musculoskeletal symptoms by the sample was $45.9 \%(n=18)$, given these workers reported the presence of pain, tingling and numbness in the body over the past 12 months, with greater involvement in lumbar $(10.5 \%)$. Also, $15.8 \%(n=6)$ reported impediment to perform normal activities in the last 12 months as a result of these problems, as well as consulted health professional in the last 12 months by musculoskeletal problems and had a musculoskeletal problem in the past seven days. There were significant prevalence of self-reported musculoskeletal disorders among the agents of urban cleaning. The scenario requires interventions in the field of health promotion at work.

Keywords: Urban Cleaning. Cumulative Trauma Disorders. Prevalence.

\section{INTRODUÇÃO}

"Como centralidade da vida humana, o trabalho, compreendido em atividades produtivas e improdutivas, permeia as relações carregadas de atribuições e responsabilidades objetivas, tanto quanto de cargas subjetivas, traz consequências diretas" para a vida do trabalhador (FORATTINI; LUCENA, 2015, p. 33). Deste modo, o trabalho faz parte do constructo humano, mas caso seja executado em condições inapropriadas pode gerar doenças diversas (BARROS; HONÓRIO, 2015; LOPES et al., 2012).

Pode-se afirmar, portanto, que a relação entre trabalho e adoecimento sempre esteve presente na história evolutiva do ser humano (ANTUNES; PRAUN, 2015; CLOT, 2011; LOPES et al., 2012; ROCHA; MENDES; MORRONE, 2012, SELIGMAN-SILVA, 2011; SILVA et al., 2014). Contudo, os autores Antunes e Praun (2015, p.410) discorrem que "com a produção em massa e a ampliação do controle e intensificação do trabalho, proporcionado pela expansão do taylorismo-fordismo, novas formas de acidentes e adoecimentos com nexo laboral passaram a fazer parte do cotidiano do trabalho".

Em decorrência de condições laborais precárias há o desvencilhar de adoecimentos com nexo laboral, com significativo aumento das lesões osteomusculares e dos transtornos mentais (ANTUNES; PRAUN, 2015). Estas lesões correspondem a "uma síndrome que vem provocando sequelas irreversíveis aos trabalhadores que podem implicar invalidez permanente. A dor e a fragilidade nos membros ou na coluna podem se tornar crônicas e impossibilitar até mesmo a realização das tarefas mais simples e banais do cotidiano" (MORAES; BASTOS, 2013, p. 3). 
Rocha; Mendes; Morrone (2012) e Baptista; Merighi; Silva (2011) reforçam a estreita relação entre o processo laboral e o surgimento de lesões osteomusculares. Para os autores supraditos, inicialmente, estas lesões eram restritas a poucos grupos ocupacionais, mas atualmente parece apresentar caráter epidêmico entre as inúmeras categorias profissionais em decorrência da implementação de tecnologias de automação e de novos modelos de gestão que exigem demasiadamente da força de trabalho, gerando desgaste do sistema osteomioarticular do operariado.

Sobre a assertiva, Harvey (2013) e Seligman-Silva (2011) enfatizam que a conformação outrora especificada reflete as reais condições de exploração do trabalhador, as quais incidem na precarização do trabalho.

Neste cenário, as condições insalubres são visíveis durante o exercício profissional dos agentes da limpeza urbana, popularmente conhecidos como garis. A labuta deste grupo de trabalhadores é caracterizada precariedade, as quais tornam o processo laboral um gerador de adoecimento (LUZ, 2012). Lopes et al. (2012) e Luz (2012) dizem que o processo de trabalho dos garis envolve um conjunto de atividades específicas, como varrição, capinação, roçada, raspação de resíduos, acondicionamento do lixo público e seu recolhimento, dentre outros. Portanto, o desempenho destas tarefas cotidianas os expõe a riscos ambientais como os físicos, químicos, ergonômicos, biológicos, mecânicos e psicossociais (CARDOSO et al., 2014; DARTORA; SANTOS, 2014; HANSELL et al., 2014; NASCIMENTO et al., 2014; OLIVEIRA; SILVA, 2015; PATARO; FERNANDES, 2014; PINHO; NEVES, 2010; PORTA et al., 2009; SOUSA, V. et al., 2015; VELLOSO; GUIMARÃES, 2013).

"Cabe ressaltar que os riscos mencionados não agem sobre o trabalhador de forma isolada. Assim, o corpo do trabalhador interage com os diversos riscos existentes, podendo adoecer e sofrer acidentes" (VELLOSO; GUIMARÃES, 2013, p. 249). Como outrora mencionado, o lidar diário com os mencionados agentes conduz ao adoecimento destes profissionais e entre os agravos merece destaque os distúrbios osteomusculares relacionados ao trabalho (DORT's).

Pesquisadores apontam a significativa prevalência de DORT's entre os garis (CARDOSO et al., 2014; DARTORA; SANTOS, 2014; KUIJER; SLUITER; FRINGSDRESEN, 2010; NAVA, 2012; PATARO; FERNANDES, 2014; PEREIRA, 2011; BALLESTEROS; ARRANGO; URREGO, 2008). Tais agravos decorrem da intensificação da jornada de trabalho, dos movimentos repetitivos, de esforços 
físicos excessivos, posturas inapropriadas, trabalho muscular estático por várias horas diárias, pressão mecânica sobre segmentos corpóreos, impactos e vibrações (ASSUNÇÃO; VILELA, 2009; PURIENE et al., 2008; VELLOSO; GUIMARÃES, 2013).

Pelas conjecturas e questionamentos desta investigação: qual a prevalência autorreferida de distúrbios osteomusculares entre agentes da limpeza urbana? Partindo da resposta a indagação se reconhece que os estudos seccionais ou transversais têm relevância ímpar, pois permitem descrever o que ocorre em determinado grupo em período temporal específico, auxiliando o processo de tomada de decisão posterior quanto ao planejamento de ações no campo da promoção de saúde (CURCI et al., 2013; LAZZARI; REIS, 2011).

O presente artigo tem por objetivo avaliar a prevalência autorreferida de distúrbios osteomusculares entre os trabalhadores da limpeza urbana. Adicionalmente, busca-se verificar a existência de correlações entre os distúrbios osteomusculares e as variáveis sociais e demográficas dos trabalhadores.

\section{MATERIAIS E MÉTODOS}

Estudo descritivo, de prevalência com abordagem quantitativa, tendo sido realizado no município de Patos - $\mathrm{PB}$, localizado no sertão paraibano, a qual possui uma população de 101 mil habitantes. A cidade conta com um serviço de limpeza urbana terceirizado, o qual possui 55 garis.

Destes trabalhadores, apenas 38 (60,1\% do universo de pesquisa) participaram da pesquisa. O número amostral foi estabelecido mediante amostra não probabilística intencional determinada conforme critérios de inclusão e exclusão. Incluíram-se os agentes de limpeza pública do município sindicalizados, os quais estavam em pleno estado de saúde física e mental e aqueles que não estavam fazendo qualquer tipo de tratamento psicológico ou medicamentoso durante o período de coleta de dados (setembro a outubro de 2015). Excluíram-se os garis que atuavam na atividade há menos de 12 meses e aqueles em licença médica durante a pesquisa.

Foram aplicados dois questionários. O primeiro, elaborado pela pesquisadora e submetido à testagem piloto, contemplou variáveis sociais, demográficas, 
profissionais e clínicas dos pesquisados. O segundo instrumento, o Questionário Nórdico de Sintomas Osteomusculares (QNSO) elaborado por Kuorinka et al. (1987), validado por Barros; Alexandre (2003) e desenvolvido com o intento de padronizar a mensuração de relatos osteomusculares e proporcionar a comparação dos resultados entre outros. O QNSO foi utilizado para identificar a partir das informações fornecidas pelos trabalhadores, as partes do corpo em que os trabalhadores sentiram dor, formigamento ou dormência causada pela atividade laboral, nos últimos 12 meses e os sete dias precedentes à entrevista, bem como verificada os relatos de afastamento das atividades rotineiras no último ano.

A coleta de dados foi realizada entre os meses de setembro a outubro de 2015, após a aprovação do projeto pelo Comitê de Ética em Pesquisa das Faculdades Integradas de Patos, CAEE 45606815.0.0000.5181/ Número do Parecer: 1.211.722, conforme preconiza a Resolução 466/12 referente à pesquisa envolvendo seres humanos (BRASIL, 2013). Durante a coleta de dados, os pesquisados foram esclarecidos quanto aos objetivos do estudo e elucidadas as dúvidas referentes aos questionários a serem aplicados. Posteriormente, foi entregue o Termo de Consentimento Livre e Esclarecido e após sua decisão voluntária em participar do estudo, destinou-se 20 minutos para 0 autopreenchimento dos instrumentos de coleta de dados.

Os dados foram analisados no Statistical Package for the Social Sciences (SPSS), versão 21. Foi utilizada a estatística descritiva (média, desvio padrão, mediana, valores mínimos e máximos, frequência e porcentagem) e a análise estatística inferencial por meio dos métodos não paramétricos - o coeficiente de correlação de Spearman e o coeficiente de correlação bisserial por ponto, ao nível de $5 \%$ de probabilidade $(p \leq 0,05)$, respectivamente.

\section{RESULTADOS}

A tabela 1 mostra a descrição dos dados ocupacionais e tempo médio que exerce a função. Verifica-se que dos 38 trabalhadores que participaram da pesquisa, a maioria são coletores de lixo $65,8 \%(n=25)$, metade $50 \%(n=19)$ trabalha no turno da manhã e apenas $2,6 \%(n=1)$ faz hora extra de oito horas; $44,7 \%(n=17)$ relataram 
se sentirem cansados após a jornada de trabalho, todos $100 \%(n=38)$ disseram que gostam do trabalho e $13,2 \%(n=5)$ tiveram afastamento nos últimos três meses.

Em relação ao tempo médio que exerce a função, os profissionais possuem em média 43,43 meses de trabalho $( \pm 45,48)$, com mínimo de 1,2 meses e máximo de 192 meses.

Tabela 1 - Descrição dos dados ocupacionais e tempo médio que exerce a função

\begin{tabular}{cccc}
\hline Variáveis & F & $\%$ \\
\hline Atividade profissional & Varrição & 13 & 34,2 \\
& Coleta de Lixo & 25 & 65,8 \\
Turno de trabalho & Manhã & 19 & 50,0 \\
& Tarde & 9 & 23,7 \\
Faz hora extra & Noite & 10 & 26,3 \\
Quantas & Sim & 1 & 2,6 \\
Após a jornada de trabalho se & Não & 37 & 97,4 \\
sente fisicamente cansado & Oito & 1 & 100 \\
Gosta do trabalho & Sim & 17 & 44,7 \\
Ficou afastado nos últimos 3 & Não & 21 & 55,3 \\
& Sim & 38 & 100,0 \\
meses & Não & 0 & 0,0 \\
& Sim & 5 & 13,2 \\
& Não & 33 & 86,8 \\
Motivo & Consulta médica & 1 & 20 \\
Média & Extração de dente & 1 & 20 \\
Desvio padrão & Férias & 1 & 20 \\
Mediana & Fratura no tornozelo & 1 & 20 \\
Mínimo & Queda de moto & 1 & 20 \\
Máximo & Tempo que exerce a função (meses) & \\
\hline
\end{tabular}

De acordo com a tabela 2 observa-se a descrição dos trabalhadores que tiveram problemas como dor, formigamento, dormência no corpo nos últimos 12 meses e verifica-se que o problema mais frequente foi na região lombar $(34,2 \%$; $n=13)$, seguida na região do pescoço e do dorso, $18,4 \%(n=7)$, respectivamente.

Ressalta-se que os trabalhadores impedidos a realizar as atividades normais nos últimos 12 meses foram afastados e a razão mais comum foi os problemas nos joelhos com $7,9 \%(n=3)$. 
Tabela 2 - Descrição dos que tiveram problemas como dor, formigamento, dormência no corpo de quem foi impedido de realizar atividades normas nos últimos 12 meses

\begin{tabular}{lcccc}
\hline Regiões anatômicas & $\begin{array}{c}\text { Descrição dos que tiveram } \\
\text { problemas como dor, } \\
\text { formigamento, dormência no } \\
\text { corpo nos últimos }\end{array}$ & $\begin{array}{c}\text { 12 meses } \\
\text { impedido de realizar atividades }\end{array}$ & $\begin{array}{c}\text { Descricao de quem foi } \\
\text { normas nos últimos 12 meses }\end{array}$ \\
\cline { 2 - 5 } Pescoço & $\mathbf{F}$ & $\%$ & $\mathbf{F}$ & $\%$ \\
Ombros & 7 & 18,4 & 2 & 5,3 \\
Região Dorsal & 6 & 15,8 & 2 & 5,3 \\
Cotovelos & 7 & 18,4 & 2 & 5,3 \\
Antebraço & 2 & 5,3 & 1 & 2,6 \\
Região Lombar & 3 & 7,9 & 1 & 2,6 \\
Punhos/Mãos/Dedos & 13 & 34,2 & 2 & 5,3 \\
Quadris e Coxas & 5 & 13,2 & 2 & 5,3 \\
Joelhos & 4 & 10,5 & 1 & 2,6 \\
Tornozelos / Pés & 4 & 10,5 & 3 & 7,9 \\
\hline & 6 & 15,8 & 2 & 5,3 \\
\hline
\end{tabular}

De acordo com o exposto na tabela 3 observa-se que 10,5\% ( $n=4)$ dos trabalhadores tiveram problemas na região lombar e 5,3\% $(n=2)$ por dores nos joelhos, nos últimos 12 meses. E para aqueles que tiveram algum tipo de problema osteomuscular nos últimos sete dias, verifica-se que apenas 10,5\% $(n=4)$ relataram problemas na região lombar.

Tabela 3 - Descrição de quem consultou profissional de saúde nos últimos 12 meses por problemas osteomusculares e do número de trabalhadores que tiveram algum problema nos últimos sete dias

\begin{tabular}{lcccc}
\hline Regiões anatômicas & \multicolumn{2}{c}{$\begin{array}{c}\text { Com consulta ao profissional de } \\
\text { saúde nos últimos } \\
\text { problemas osteomusculares }\end{array}$} & $\begin{array}{c}\text { Trabalhadores que tiveram } \\
\text { algum problema nos últimos } \\
\text { sete dias }\end{array}$ \\
\cline { 2 - 5 } & $\mathbf{F}$ & $\%$ & $\mathbf{F}$ & $\%$ \\
\hline Pescoço & 1 & 2,6 & 1 & 2,6 \\
\hline Ombros & 0 & 0,0 & 1 & 2,6 \\
\hline Região Dorsal & 1 & 2,6 & 2 & 5,3 \\
\hline Cotovelos & 0 & 0,0 & 0 & 0,0 \\
\hline Antebraço & 0 & 0,0 & 0 & 0,0 \\
\hline Região Lombar & 4 & 10,5 & 4 & 10,5 \\
\hline Punhos/Mãos/Dedos & 0 & 0,0 & 0 & 0,0 \\
\hline Quadris e Coxas & 0 & 0,0 & 0 & 0,0 \\
\hline Joelhos & 0 & 5,3 & 1 & 2,6 \\
\hline Tornozelos / Pés & 2 & 0,0 & 0 & 0,0 \\
\hline
\end{tabular}

A tabela 4 mostra a prevalência de sintomas osteomusculares autorreferida pela amostra. Verifica-se que $45,9 \%(n=18)$ apresentaram dor, formigamento, dormência no corpo e $15,8 \%(n=6)$ relataram impedimento de realizar atividades normais nos últimos 12 meses por tal sintomatologia. Observou-se ainda que aqueles que consultaram o profissional de saúde nos últimos 12 meses por tais 
problemas e que tiveram alguma intercorrência osteomuscular nos últimos sete dias apresentaram $15,8 \%(n=6)$, respectivamente.

Tabela 4 - Descrição da prevalência autorreferida de sintomas osteomusculares

\begin{tabular}{lcc}
\hline Presença de sintomas osteomusculares & F & $\%$ \\
\hline Dor, formigamento, dormência no corpo nos últimos 12 meses & 18 & 45,9 \\
$\begin{array}{l}\text { Impedido de realizar atividades normas nos últimos 12 meses por problemas } \\
\text { osteomusculares }\end{array}$ & 6 & 15,8 \\
$\begin{array}{l}\text { Consultou profissional de saúde nos últimos 12 meses por problemas } \\
\text { osteomusculares }\end{array}$ & 6 & 15,8 \\
$\begin{array}{l}\text { Trabalhadores que tiveram algum problema nos últimos sete dias por } \\
\text { problemas osteomusculares }\end{array}$ & 6 & 15,8 \\
\hline
\end{tabular}

De acordo com o exposto na tabela 5 observa-se que as correlações entre os sintomas osteomusculares com os dados sociais, demográficos e profissionais, a idade se correlacionou significativamente e positivamente $(\rho=0,34 ; p<0,05)$ com número de vezes que se consultou o profissional de saúde nos últimos 12 meses, sugerindo aumento nas consultas com a elevação da idade. Outra correlação estatisticamente significativa e positiva $(\rho=0,35 ; p<0,05)$ foi entre o número de filhos e as vezes que apresentou algum problema nos últimos sete dias. O tempo de exercício da função também se correlacionou positivamente e significativamente ( $\rho=$ 0,52; $p<0,01)$ com o número de vezes que teve problemas como dor, formigamento, dormência no corpo nos últimos 12 meses.

Também, os resultados mostram correlação significativa e positiva $(\rho=0,47$; $p<0,01)$ entre aqueles trabalhadores que após o trabalho se sentiam cansados fisicamente e o número de vezes que teve problemas como dor, formigamento, dormência no corpo nos últimos 12 meses, bem como com o número de vezes que teve algum problema nos últimos sete dias $(\rho=0,33 ; p<0,05)$. 
Tabela 5 - Correlação entre os sintomas osteomusculares com os dados sociais, demográficos e profissionais (variáveis contínuas)

\begin{tabular}{|c|c|c|c|c|c|c|c|c|c|}
\hline \multirow[b]{2}{*}{ Variáveis } & \multicolumn{4}{|c|}{$\begin{array}{c}\text { Dados sociais e } \\
\text { demográficos }\end{array}$} & \multicolumn{5}{|c|}{ Dados profissionais } \\
\hline & $\begin{array}{l}\stackrel{0}{0} \\
\frac{\pi}{0} \\
\text { 은 }\end{array}$ & 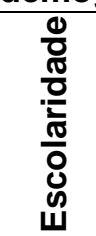 & 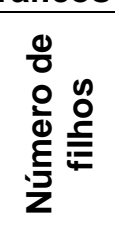 & $\begin{array}{l}\frac{\pi}{0} \\
\frac{\sigma}{\Phi} \\
\mathbb{\alpha}\end{array}$ & 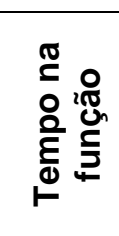 & $\begin{array}{l}\frac{0}{0} \\
\frac{0}{0} \\
\frac{\pi}{0} \\
\frac{0}{2} \\
\frac{0}{2} \\
\frac{0}{2} \\
\frac{0}{2}\end{array}$ & 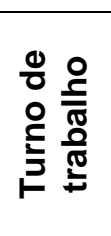 & 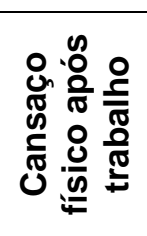 & 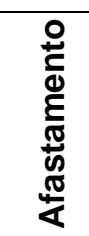 \\
\hline $\begin{array}{l}\text { Número de vezes que teve } \\
\text { problemas } \quad \text { como } \\
\text { formigamento, dor, } \\
\text { corpo nos últimos } 12 \text { meses }\end{array}$ & 0,04 & $\overline{-}, 26$ & 0,23 & 0,16 & $0,52^{* *}$ & 0,18 & $-0,18$ & $0,47^{* *}$ & 0,05 \\
\hline $\begin{array}{l}\text { Número de vezes que foi } \\
\text { impedido de realizar atividades } \\
\text { normas nos últimos } 12 \text { meses }\end{array}$ & $-0,00$ & $\overline{-}, 18$ & 0,02 & $\overline{0}, 11$ & 0,16 & 0,12 & 0,01 & 0,19 & 0,26 \\
\hline $\begin{array}{l}\text { Número de vezes que consultou } \\
\text { profissional de saúde nos } \\
\text { últimos } 12 \text { meses }\end{array}$ & $0,34^{*}$ & 0,19 & 0,12 & 0,01 & 0,20 & 0,07 & $-0,01$ & 0,24 & 0,14 \\
\hline $\begin{array}{l}\text { Número de vezes que teve } \\
\text { algum problema nos últimos } \\
\text { sete dias }\end{array}$ & 0,09 & $\overline{-}, \overline{08}$ & $0,35^{*}$ & $\overline{0,09}$ & 0,26 & 0,01 & $-0,24$ & $0,33^{*}$ & 0,10 \\
\hline
\end{tabular}

Teste de correlação bisserial por ponto

Atividade profissional - 1 varrição; 2 coleta de lixo; Turno do trabalho - 1 manhã; 2 tarde; 3 noite; Hora extra - 1 não; 2 sim; Cansaço físico após trabalho - 1 não; 2 sim; Ficou afastado - 1 não; 2 sim.

${ }^{*} p \leq 0,05 ;{ }^{* *} p \leq 0,01$.

A tabela 6 mostra as correlações entre os sintomas osteomusculares com os dados clínicos, a hipertensão e verificou-se correlação estatisticamente significativa apenas com a hipertensão. Os hipertensos realizaram mais consultas ao profissional de saúde nos últimos 12 meses; e maior número de vezes que teve algum problema nos últimos sete dias.

Tabela 6 - Correlação dos sintomas osteomusculares com os dados clínicos (variáveis categóricas)

\begin{tabular}{|c|c|c|c|c|c|}
\hline Variáveis & Hipertenso & Diabético & $\begin{array}{l}\text { Atividade } \\
\text { física }\end{array}$ & Fumante & Etilista \\
\hline $\begin{array}{l}\text { Número de vezes que teve problemas } \\
\text { como dor, formigamento, dormência no } \\
\text { corpo nos últimos } 12 \text { meses }\end{array}$ & 0,30 & $-0,10$ & $-0,22$ & $-0,16$ & 0,18 \\
\hline $\begin{array}{l}\text { Número de vezes que foi impedido de } \\
\text { realizar atividades normas nos últimos } 12 \\
\text { meses }\end{array}$ & 0,17 & $-0,05$ & $-0,19$ & $-0,17$ & 0,28 \\
\hline $\begin{array}{l}\text { Número de vezes que consultou } \\
\text { profissional de saúde nos últimos } 12 \\
\text { meses }\end{array}$ & $0,81^{\star \star}$ & $-0,06$ & $-0,05$ & $-0,14$ & 0,08 \\
\hline $\begin{array}{l}\text { Número de vezes que teve algum } \\
\text { problema nos últimos } 7 \text { dias }\end{array}$ & $0,47^{* *}$ & $-0,06$ & 0,01 & $-0,14$ & 0,27 \\
\hline
\end{tabular}

Fonte: Dados de Pesquisa, 2015.

Teste de correlação bisserial por ponto

Hipertenso - 1 não; 2 sim; Diabético - 1 não; 2 sim; Atividade física - 1 não; 2 sim; Fumante - 1 não; 2 sim; Etilista - 1 não; 2 sim

${ }^{*} p \leq 0,05 ;{ }^{* \star} p \leq 0,01$. 


\section{DISCUSSÃO}

A presença de dor, formigamento e dormência, ou seja, de sintomatologia osteomuscular é relatada em vários grupos de trabalhadores, tanto em pesquisas nacionais (ALENCAR; CAVALCANTI; MONTREZOR, 2013; ALMEIDA et al., 2012; LIMA et al., 2015; LUNA; SOUZA, 2014; MANGO et al., 2012; MARQUESI; ROVIDA; GARBIN, 2013; MEEREIS et al., 2013; OLIVEIRA et al., 2015; OLIVEIRA; SOUZA, 2015; SOUSA, M. et al., 2015; SOUZA; GOMES NETO, 2015; VASCÃO et al., 2014) quanto internacionais (COENEN et al., 2013; HARVIN, 2014; KIERMAYER et al., 2011; KIM; ROH, 2014; KOZAK et al., 2014; LEE et al., 2013; MAHMUD et al., 2011; OGLE; RUBIN; SIEGLER, 2014; SPREEUWERS et al., 2011).

Contudo, estudos sobre DORT'S direcionados aos agentes da limpeza urbana ainda são poucos (CARDOSO et al., 2014; DARTORA; SANTOS, 2014; ENGLEHARDT; FLEMING; BEAN, 2003; MEHRDAD et al., 2008; MEDERIROS et al., 2014; NASCIMENTO et al., 2014; PATARO; FERNANDES, 2014; BALLESTEROS; ARRANGO; URREGO, 2008), não refletindo a real situação e/ou dimensão da problemática que compadecem.

Apesar disto, os resultados encontrados confirmaram a existência de dor, formigamento e dormência em algum segmento corporal dos agentes da limpeza urbana, pois quase a metade $(45,9 \%)$ relatou queixas osteomusculares em alguma região do corpo nos últimos 12 meses. Tal resultado era esperado, pois Kuijer; Sluiter; Frings-Dresen (2010) ao realizaram uma revisão de literatura em Amsterdã na Holanda, com o propósito de identificar lesões em trabalhadores da coleta de lixo, encontraram evidências de risco para doenças e musculoesqueléticas.

Também, as características do trabalho desenvolvido pelos agentes de limpeza corroboram para o aparecimento de queixas osteomusculares. O processo de trabalho é considerado fisicamente pesado, trabalho muscular estático por várias horas diárias, com jornadas exaustivas, repetições de movimentos, longos períodos de pé, posturas inapropriadas, entre outros aspectos (ASSUNÇÃO; VILELA, 2009; PURIENE et al., 2008; VELLOSO; GUIMARÃES, 2013). Sobre a afirmativa, quase a metade dos pesquisados desta investigação afirmam estar cansados após a labuta diária e os resultados mostraram haver correlação significativa e positiva entre aqueles trabalhadores que após o trabalho se sentiam cansados fisicamente e o 
número de vezes que apresentaram problemas como dor, formigamento, dormência no corpo nos últimos 12 meses, bem como com o número de vezes que teve algum problema nos últimos sete dias.

Contribuindo para o surgimento de DORT's, soma-se o tempo de trabalho na ocupação realizando a mesma atividade, que neste estudo foi de $43,43( \pm 45,48)$. Neste estudo, o tempo de exercício da função se correlacionou positivamente e significativamente $(\rho=0,52 ; p<0,05)$ com o número de vezes que teve problemas como dor, formigamento, dormência no corpo nos últimos 12 meses. Cardoso et al. (2014) mediante pesquisa com coletores de lixo constaram que entre os trabalhadores que relataram queixas musculoesqueléticas, 94,5\% não possuíam qualquer dor antes de exercerem a atividade de coleta de lixo.

Por conseguinte, afirma-se que a prevalência de DORT's autorreferida entre o grupo estudo foi considerada elevada e os achados se assemelham percentualmente com a pesquisa de Nascimento et al. (2014), desenvolvida com trabalhadores garis da cidade de Lagarto, Sergipe. Os pesquisadores identificaram que as DORT's incidiram sobre $40,7 \%$ dos pesquisados.

Contudo, Cardoso et al. (2014), com o objetivo de verificar a prevalência de distúrbios osteomusculares em coletores de lixo de Pelotas e Rio Grande, cidades de porte médio do sul do Brasil, encontraram prevalência superior de DORT's $(88,2 \%)$ entre os 127 coletores de lixo que participam do estudo. Englehardt; Fleming; Bean (2003), em investigação realizada na Flórida, Estados Unidos, identificaram prevalência de $75 \%$ de lesões osteomusculares no último ano entre os coletores de lixo sólido domiciliar.

Pataro; Fernandes (2014) identificaram prevalência de dor ou desconforto nos últimos 12 meses em 77,4\% da amostra. E Mehrdad et al. (2008), a partir de investigação com coletores de lixo na cidade de Teerã, no Iran, constaram uma prevalência de DORT's de 65\%.

Quanto a região do corpo mais acometida, a lombar foi a mais ressaltada entre o grupo pesquisado, contudo, a região do pescoço e do dorso também apresentaram destaque. Dados compatíveis foram encontrados nos estudos de Mederiros et al. (2014) e Pataro; Fernandes (2014).

Com as evidências, nota-se que a região da coluna é um dos segmentos corporais mais prejudicados, posteriormente as extremidades do corpo e parte 
inferior (MEDERIROS et al., 2014). Sobre a região lombar, alvo das principais queixas dos trabalhadores desta pesquisa em Patos, Paraíba, Grandjean (2005) e lida (2005) enfatizam que intervenções ergonômicas precisam ser implementadas com urgência, pois os problemas de coluna além de causarem desconforto e de serem dolorosos, podem reduzir a mobilidade e a vitalidade do trabalhador.

Adicionalmente há que se reforçar que, embora a lombar tenha sido a região mais afetada, outros segmentos também foram apontados neste estudo, condição também observada nas pesquisas de Cardoso et al. (2014), Harvin (2014); Kim; Roh (2014), Ogle; Rubin; Siegler (2014), Oliveira et al. (2015), Oliveira; Souza (2015); Souza; Gomes Neto (2015), Vascão et al. (2014).

Pelas exposições, assevera-se que a coleta de lixo é uma atividade laboral de significativa importância para a sociedade, contudo, o processo de trabalho dos agentes da limpeza urbana conduz à vulnerabilidade a prejuízos à saúde decorrentes do labor (MEDERIROS et al., 2014).

Pela prevalência de DORT's considerada elevada neste estudo e em outros com percentual variando entre $40,7 \%$ a $88,2 \%$, poucos trabalhadores buscaram consultar um profissional de saúde nos últimos 12 meses por problemas osteomusculares. $\mathrm{O}$ achado faz refletir sobre as razões pelas quais tais profissionais permanecem com a sintomatologia osteomuscular sem buscar terapêutica. Portanto, talvez decorra do medo de ficar desempregado e sem conseguir manter o sustento da família. Santos et al. (2009, p. 206) afirmam que "os garis omitem suas queixas aos gerentes, temendo a demissão".

Quanto ao correlato entre os sintomas osteomusculares com os dados sociais, demográficos e profissionais, constatou-se que com o aumentar da idade também se eleva o número de consultas ao profissional de saúde. Bastos; Harzheim; Sousa (2014), sobre a relevância e fatores associados à consulta médica entre adultos de uma comunidade de baixa renda do Sul do Brasil, constataram maior busca pelo profissional de saúde com o aumentar da faixa etária. Também, Rosa Filho; Fassa; Paniz (2008), em pesquisa sobre fatores associados à continuidade interpessoal na atenção à saúde também identificaram maior realização de consultas com o aumento da idade.

O fato outrora elucidado também pode ter relação com a percepção da doença, Spreeuwers et al. (2011) constaram que indivíduos mais velhos tem pior 
perceção sobre severidade de algum agravo (doenças ocupacionais) e isto compromete sua vida diária.

Outra correlação estatisticamente significativa e positiva ocorreu entre 0 número de filhos e as vezes que apresentou algum problema osteomuscular nos últimos sete dias, corroborando com os estudos de Luna; Souza (2014). Os autores identificaram associação estatisticamente relevante entre sintomas osteomusculares e número de filhos. Assim sendo, os mencionados pesquisadores atribuíram o resultado as despesas com a família, a qual é muito maior a depender do número de filhos. Ressalta-se ainda que o estudo realizado pelo Instituto de Brasileiro de Geográfia e Estatística (IBGE, 2012) mostra que 30\% das mulheres garis respondem sozinhas pela manutenção econômica, cuidados com a casa e com filhos. Nesse cenário percebesse-se a formação de famílias monoparentais, onde as mulheres são responsáveis pelo sustento da família e as obrigações do lar reforçando então a dupla jornada, o que as deixam mais susceptíveis ao acometimento por DORT's.

Contemplando o correlato entre sintomatologia osteomuscular e os dados clínicos, os hipertensos realizaram mais consultas ao profissional de saúde nos últimos 12 meses; e maior número de vezes que teve algum problema nos últimos sete dias. Este achado pode ter relação com o fato de que entre as cinco primeiras causas de adoecimento da população beneficiada pela previdência social, três referem-se intrinsecamente aos grupos de Doenças Crônicas Não Transmissíveis: as doenças osteomusculares, as do aparelho circulatório (como a hipertensão) e os transtornos mentais (MOURA; CARVALHO; SILVA, 2007). Carrolo et al. (2013) numa comunidade de coletores de lixo também constaram entre os problemas mais prevalentes a hipertensão e os problemas musculares.

Spreeuwers et al. (2011), em estudo sobre problemas osteomusculares relacionadas com o trabalho, constatou que aqueles indivíduos com alguma doença têm maior comprometimento funcional e redução na qualidade de vida, o que pode estar relacionado inclusive com a sintomatologia osteomuscular.

Finaliza-se a discussão afirmando que inexistem muitos estudos publicados no Brasil e no mundo com o propósito de avaliar a saúde ocupacional de agentes da limpeza pública, fato que dificultou o processo de discussão deste artigo. Adicionalmente, como outra limitação destaca-se o percurso metodológico adotado e 
o tamanho da amostra. Mesmo assim, os achados desta abordagem assemelhamse as evidências de outras pesquisas nacionais e internacionais.

\section{CONCLUSÃO}

Constatou-se significativa prevalência de distúrbios osteomusculares autorreferida entre os agentes da limpeza urbana, com maior acometimento da região lombar. Outro dado importante e que necessita de atenção e intervenção foi o fato daqueles trabalhadores que relataram cansados fisicamente após a jornada de trabalho correlaciona-se positivamente com a dor, formigamento, dormência no corpo nos últimos 12 meses e últimos sete dias, ou seja, com a sintomatologia osteomuscular.

Também, o tempo na função associou-se ao relato de sintomatologia osteomuscular no último ano. Pelos achados, estratégias preventivas apropriadas são necessárias, sendo imprescindível a instituição de medidas no ambiente de trabalho para minimizar seus efeitos. Sugere-se a adoção da Análise Ergonômica do Trabalho para verificação e a adoção de práticas complementares para minimização dos impactos sobre o sistema osteomioarticular, melhorando a qualidade de vida dos trabalhadores.

\section{REFERÊNCIAS}

ALENCAR, M. C. B.; CAVALCANTI, T. A.; MONTREZOR, J. B. Condições de trabalho em uma cozinha industrial e distúrbios osteomusculares de trabalhadores. Cad. Ter. Ocup. UFSCar, São Carlos, v. 21, n. 1, p. 155-62, 2013.

ALMEIDA, M. C. V. et al. Prevalência de doenças musculoesqueléticas entre trabalhadores portuários avulsos. Rev. Latino-Am. Enfermagem, Ribeirão Preto, v. 20, n. 2, p. 243-250, abr.2012. https://doi.org/10.1590/S0104-11692012000200005

ANTUNES, R.; PRAUN, L. A sociedade dos adoecimentos no trabalho. Serv. Soc. Soc., São Paulo, n. 123, p. 407-27, jul./set. 2015.

ASSUNÇÃO, A. Á.; VILELA, L. V. Lesões por esforços repetitivos: guia para profissionais de saúde. Piracicaba: CEREST, 2009.

BALLESTEROS, V. L.; ARANGO, Y. L. L.; URREGO, Y. M. C. Condiciones de salud y de trabajo informal en recuperadores ambientales del área rural de Medellín, Colombia. Revista de Saúde Pública, v. 46, n. 5, p. 866-74, 2012. https://doi.org/10.1590/S0034-

$\underline{89102012000500014}$ 
BAPTISTA, P. C. P.; MERIGHI, M. A. B.; SILVA, A. Angústia de mulheres trabalhadoras de enfermagem que adoecem por distúrbios osteomusculares relacionados ao trabalho.

Revista Brasileira de Enfermagem. v. 64, n. 3, p. 438-44, 2011.

https://doi.org/10.1590/S0034-71672011000300005

BARROS, E. N. C.; ALEXANDRE, N. M. C. Cross-Cultural Adaptation of the Nordic Musculokeletal Questionnaire. Int Nurs Rev., v. 50, n. 2, p. 101-08, 2003.

https://doi.org/10.1046/j.1466-7657.2003.00188.x

BARROS, N. M. G. C.; HONÓRIO, L. C. Riscos de adoecimento no trabalho de médicos e enfermeiros em um hospital regional mato-grossense. REGE, São Paulo, v. 22, n. 1, p. 21 39, jan./mar. 2015. https://doi.org/10.5700/rege549

BASTOS, G. A. N.; HARZHEIM, E.; SOUSA, A. I. Prevalência e fatores associados à consulta médica entre adultos de uma comunidade de baixa renda do Sul do Brasil.

Epidemiol. Serv. Saúde, Brasília, v. 23, n. 3, p. 409-20, set. 2014.

BRASIL. Conselho Nacional de Saúde. Resolução 466/12. Dispõe sobre as diretrizes e normas regulamentadoras de pesquisas envolvendo seres humanos. Brasília: CNS, 2013.

CARDOSO, R. K. et al. Osteomuscular disorders and associated factors among solid waste collectors of two middle-sized cities from the South of Brazil. Rev Dor, São Paulo, v. 15, n. 1, p. 13-6, 2014. https://doi.org/10.5935/1806-0013.20140004

CLOT, Y. Clínica do trabalho e clínica da atividade. In: BENDASSOLLI, P. F.; SOBOLL, L. A. (Orgs.). Clínicas do trabalho: novas perspectivas para compreensão do trabalho na atualidade. São Paulo: Atlas, 2011.

COENEN, P. et al. Cumulative Low Back Load at Work as a Risk Factor of Low Back Pain: A Prospective Cohort Study. J Occup Rehabil., v. 23, n. 1, p. 11-8, Mar. 2013.

https://doi.org/10.1007/s10926-012-9375-z

CURCI, K. A. et al. Promoção da saúde e prevenção de riscos e doenças na Saúde Suplementar: um breve histórico. Mundo Saúde, v. 37, n. 2, p. 230-40, 2013.

DARTORA, J.; SANTOS, M. V. Cinesioterapia laboral preparatória para trabalhadores de limpeza e urbanização de uma empresa do Vale do Taquari/RS. Revista Destaques

Acadêmicos, v. 6, n. 3, p. 1-10, 2014.

ENGLEHARDT, J. D.; FLEMING, L. E.; BEAN, J.A. Analytical predictive Bayesian assessment of occupational injury risk: municipal solid waste collectors. Risk Anal., v. 23, n. 5, p. 917-27, 2003. https://doi.org/10.1111/1539-6924.00369

FERNANDES, R. C. P Heavy physical work and low back pain: the reality in urban cleaning. Rev Bras Epidemiol., v. 17, n. 1, p. 17-30, 2014. https://doi.org/10.1590/1415790X201400010003ENG

FORATTINI, C. D.; LUCENA, C. Adoecimento e sofrimento docente na perspectiva da precarização do trabalho. Laplage em Revista, Sorocaba, v. 01, n.2, , p.32-47, maio.- ago 2015.

GRANDJEAN, E. Manual de ergonomia: adaptando o trabalho ao homem. 5. ed. Porto Alegre: Bookman, 2005. 
HANSELL, A. et al. Occupational risk factors for chronic respiratory disease in a New Zealand population using lifetime occupational history. Journal Of Occupational And Environmental Medicine, Nova Zelândia, v. 56, n. 3, p.80-270, mar. 2014.

HARVEY, D. A condição pós-moderna. São Paulo: Loyola, 2013.

HARVIN, G. Review of Musculoskeletal Injuries and Prevention in the Endoscopy Practitioner. J Clin Gastroenterol., v. 48, n. 7, p. 590-4, Aug. 2014.

https://doi.org/10.1097/MCG.0000000000000134

INSTITUTO BRASILEIRO DE GEOGRAFIA E ESTATÍSTICA (IBGE). Pesquisa Nacional Por Amostra de Domicílios. Síntese de indicadores. 2012. Disponível em: http://www.ibge.gov.br/home/estatistica/populacao/ trabalhoerendimento/pnad2012/ Acessado em 11 mai. 2016.

IIDA, I. Ergonomia: projeto e produção. São Paulo: Edgard Blücher, 2005.

KIERMAYER, C. et al. Musculoskeletal Load in and Highly Repetitive Actions of Animal Facility Washroom Employees. J Am Assoc Lab Anim Sci., v. 50, n. 5, p. 665-74, Sep. 2011.

KIM, T.; ROH, H. Analysis of Risk Factors for Work-related Musculoskeletal Disorders in Radiological Technologists. J Phys Ther Sci., v. 26, n. 9, p. 1423-8, Sep. 2014. https://doi.org/10.1589/jpts.26.1423

KOZAK, A. et al. Self-reported musculoskeletal disorders of the distal upper extremities and the neck in german veterinarians: a cross-sectional study. PLoS One, v. 9, n. 2, p. 89362, 2014. https://doi.org/10.1371/journal.pone.0089362

KUIJER, P. P.; SLUITER, J. K.; FRINGS-DRESEN, M. H. Health and safety in waste collection: Towards evidence-based worker health surveillance. Am J Ind Med., v. 53, n. 10, p. 1040-1064, 2010. https://doi.org/10.1002/ajim.20870

KUORINKA, I. et al. Standardised Nordic questionnaires for the analysis of musculoskeletal symptoms. Applied Ergonomics, v. 18, n. 3, p. 233-37, 1987. https://doi.org/10.1016/0003$\underline{6870(87) 90010-X}$

LAZZARI, M. A.; REIS, C. B. Os coletores de lixo urbano no município de Dourados (MS) e sua percepção sobre os riscos biológicos em seu processo de trabalho. Ciência e Saúde Coletiva, Mato Grosso do Sul, v. 16, n. 8, p. 3437-42, fev.

2011. https://doi.org/10.1590/S1413-81232011000900011

LEE, H. et al. Musicians' Medicine: Musculoskeletal Problems in String Players. Clin Orthop Surg., v. 5. n. 3, p. 155-160, Sep. 2013. https://doi.org/10.4055/cios.2013.5.3.155

LIMA, J. P. et al. Prevalência de distúrbios osteomioarticulares e algias em fisioterapeutas. Rev. Saúde Públ. Santa Cat., Florianópolis, v. 8, n. 3, p. 98-108, set./dez. 2015.

LOPES, F. T. et al. O Significado do Trabalho para os Garis: um estudo sobre representações sociais. Perspectivas em Políticas Públicas, v. 5, n. 10, p. 41-69, 2012.

LUNA, J. S.; SOUZA, O. F. Sintomas osteomusculares em taxistas de Rio Branco, Acre: prevalência e fatores associados. Cad. Saúde Colet., Rio de Janeiro, v. 22, n. 4, p. 401-8, 2014. 
LUZ, L. C. A. Segurança e saúde do trabalhador em serviços de limpeza urbana: estudo de casos. 2012. 501f. Tese (Doutorado em Engenharia Civil) - Universidade Estadual de Campinas, Faculdade de Engenharia Civil, Arquitetura e Urbanismo. Campinas: [s.n.], 2012.

MAHMUD, N. et al. Ergonomic Training Reduces Musculoskeletal Disorders among Office Workers: Results from the 6-Month Follow-Up. Malays J Med Sci.. v. 18, n. 2, p. 16-26, AprJun. 2011.

MANGO, M. S. M. et al. Análise dos sintomas osteomusculares de professores do ensino fundamental em Matinhos (PR). Fisioter Mov., v. 25, n. 4, p. 785-9, out/dez. 2012. https://doi.org/10.1590/S0103-51502012000400011

MARQUESI, C.; ROVIDA, T. A. S.; GARBIN, A. J. I. Prevalência de distúrbio osteomusculares e qualidade de vida em Cirurgiões Dentistas. Arch Health Invest, v. 2, n. esp., p. 1, 2013.

MEDERIROS, I. L. et al. Avaliação de equipamentos de proteção individual: um estudo sobre os coletores de lixo domiciliar. Design \& Tecnologia, v. 8, p. 23-30, 2014.

MEHRDAD, R. et al. Musculoskletal disorders among solid waste workers. Acta Medica Iranica, v. 3, n. 46, p. 1, 2008.

MORAES, P. W. T.; BASTOS, A. V. B. As LER/DORT e os fatores psicossociaisi. Arquivos Brasileiros de Psicologia, Rio de Janeiro, v. 65, n. 1, p. 2-20, 2013.

MOURA, A. A. G.; CARVALHO, E. F.; SILVA, N. J. C. Repercussão das doenças crônicas não-transmissíveis na concessão de benefícios pela previdência social. Ciênc. saúde coletiva, Rio de Janeiro, v. 12, n. 6, p. 1661-72, dez. 2007.

NASCIMENTO, J. C. et al. Condições de saúde e trabalho dos profissionais garis em Lagarto - SE. Interface (Botucatu) [online], supl. 3, p. 1, 2014.

NAVA, C. S. A ocorrência de sintomas osteomusculares em coletores de lixo e varredores de rua de um município da região metropolitana de Campinas. 2012. 92f. Dissertação (Mestrado em Saúde Coletiva) - Universidade Estadual de Campinas, Faculdade de Ciências Médicas. Campinas: [s.n.], 2012.

OGLE, C. M.; RUBIN, D. C.; SIEGLER, I. C. Cumulative exposure to traumatic events in older adults. Aging Ment Health, v. 18, n. 3, p. 316-25, Apr. 2014.

https://doi.org/10.1080/13607863.2013.832730

OLIVEIRA, A. O.; SOUZA, S. T. M. Lesões por esforços repetitivos/distúrbios osteomusculares relacionados a atividade bancária. Sistemas \& Gestão, v. 10, p 124-32, 2015. https://doi.org/10.7177/sg.2015.v10.n1.a10

OLIVEIRA, A. S. S.; SILVA, E. M. Insegurança: os EPI's e a realidade do trabalhador parintinense. RELEM - Revista Eletrônica Mutações, v. 1, n. 1, p. 176-8, jan./jul. 2015.

OLIVEIRA, M. M. et al. Problema crônico de coluna e diagnóstico de distúrbios osteomusculares relacionados ao trabalho (DORT) autorreferidos no Brasil: Pesquisa Nacional de Saúde, 2013. Epidemiol. Serv. Saúde, Brasília, v. 24, n. 2, jun. 2015.

PEREIRA, A. L. P. Prevalência de distúrbios musculoesqueléticos em membros superiores e fatores associados em trabalhadores de limpeza urbana de Salvador, 
Bahia. 2011. 85f. Dissertação (Mestrado em Saúde, Ambiente e Trabalho) - Universidade Federal da Bahia, Faculdade de Medicina da Bahia. Salvador: [s.n.], 2011.

PINHO, L. M.; NEVES, E. B. Acidentes de trabalho em uma empresa de coleta de lixo urbano. Cad. Saúde Colet., Rio de Janeiro, v. 18, n. 2, p. 243-51, 2010.

PORTA, D. et al. Systematic review of epidemiological studies on health effects associated with management of solid waste. Environ Health, v. 23, n. 8, p. 60, 2009.

https://doi.org/10.1186/1476-069X-8-60

PURIENE, A. et al. Self-reported occupational health issues among Lithuanian dentists. Ind Health, v. 46, n. 4, p. 369-374, 2008. https://doi.org/10.2486/indhealth.46.369

ROCHA, S. R. A.; MENDES, A. M.; MORRONE, C. F. Sofrimento, distúrbios osteomusculares e depressão no contexto de trabalho: uma abordagem psicodinâmica. Estudos e Pesquisas em Psicologia, Rio de Janeiro, v. 12, n. 2, p. 379-94, 2012.

ROSA FILHO, L. A.; FASSA, A. G.; PANIZ, V. M. V. Fatores associados à continuidade interpessoal na atenção à saúde: estudo de base populacional. Cad. Saúde Pública, Rio de Janeiro, v. 24, n. 4, p. 915-25, abr. 2008.

SANTOS, M. C. O. et al. Desregulamentação do trabalho e desregulação da atividade: o caso da terceirização da limpeza urbana e o trabalho dos garis. Produção, v. 19, n. 1, p. 202-13, 2009. https://doi.org/10.1590/S0103-65132009000100013

SELIGMAN-SILVA, E. Trabalho e Desgaste Mental: o direito de ser dono de si mesmo. São Paulo. Cortez. 2011.

SILVA, B. A. M. et al. Distúrbios osteomusculares autorreferidos em funcionários de supermercado. Rev Bras Promoç Saúde, Fortaleza, v. 27, n. 1, p. 13-20, jan./mar., 2014.

SOUSA, M. N. A. et al. Musculoskeletal disorders in informal mining workers. International Archives of Medicine, v. 8, n. 183, p. 1-8, 2015.

SOUSA, V. L. et al. Estresse ocupacional e qualidade de vida de profissionais da limpeza urbana. Rev. Saúde Públ. Santa Cat., v. 8, n. 2, p. 8-20, 2015.

SOUZA, K. V. L.; GOMES NETO, M. Análise da qualidade de vida e distúrbios osteomusculares dos funcionários administrativos de um órgão público. Revista Pesquisa em Fisioterapia, v. 5, n. 3, p. 218-29, dez. 2015. https://doi.org/10.17267/2238$\underline{2704 \text { rpf.v5i3.703 }}$

SPREEUWERS, D. et al. Work-related upper extremity disorders: one-year follow-up in an occupational diseases registry. Int Arch Occup Environ Health, v. 84, n. 7, p. 789-96, Oct. 2011. https://doi.org/10.1007/s00420-011-0611-1

VASCÃO, A. M. et al. Distúrbios osteomusculares relacionados ao trabalho (DORT) nos trabalhadores de enfermagem: revisão de literatura. Revista saúde, v. 8, n. 3-4, P. 63, 2014.

VELLOSO, M. P.; GUIMARAES, M. B. L. A imagem na pesquisa qualitativa em saúde. Ciênc. saúde coletiva, v. 18, n. 1, p. 245-252, 2013. 


\section{(c) (1)}

Artigo recebido em 18/03/2016 e aceito para publicação em 27/06/2016

DOI: http://dx.doi.org/10.14488/1676-1901.v17i1.2391 